\title{
Five Nontrivial Solutions of p-Laplacian Problems Involving Critical Exponents and Singular Cylindrical Potential
}

\author{
Mohammed el Mokhtar ould el Mokhtar \\ Departement of Mathemtics, Qassim University, BO 6644, Buraidah 51452, Kingdom of Saudi Arabia
}

\begin{abstract}
In this paper, we establish the existence of at least five distinct solutions to a p-Laplacian problems involving critical exponents and singular cylindrical potential, by using the Nehari manifold, concentration-compactness principle and mountain pass theorem
\end{abstract}

Key words: Nehari manifold, concentration-compactness principle, critical Hardy-Sobolev exponent, singular cylindrical potential, mountain pass theorem, nontrivial cylindrical solution.

\section{Introduction}

In this paper, we consider the multiplicity results of nontrivial solutions of the following problem:

$$
\left\{\begin{array}{l}
-\Delta_{p} u-\mu|y|^{-p}|u|^{p^{-2}} u=h|y|^{-s}|u|^{p^{*}(s)-2} u+\lambda f|u|^{q-2} u \text { in } \mathbb{R}^{N}, y \neq 0 \\
u \in \mathcal{D}_{1}^{p}\left(\mathcal{R}^{N}\right),
\end{array}\right.
$$

where $\Delta_{p} u=\operatorname{div}\left(|\nabla u|^{p-2} \nabla u\right), 1<\mathrm{p}<\mathrm{k}, \mathrm{k}$ and $\mathrm{N}$ are integers with $\mathrm{N}>\mathrm{p}, 2<\mathrm{k}<\mathrm{N}$, $\mathcal{R}^{N}=\mathcal{R}^{k} \times \mathcal{R}^{N-k}$, the point $\mathrm{x} \in \mathcal{R}^{N}$ can be written as $\mathrm{x}=(\mathrm{y}, \mathrm{z}) \in \mathcal{R}^{k} \times \mathcal{R}^{N-k}$,

$$
-\infty<\mu<\bar{\mu}_{k, p}:=((k-p) / p)^{p}, 0<\mathrm{s}<\mathrm{p},
$$
$p^{*}(s)=p(N-s) /(N-p) \quad$ is the critical Hardy-Sobolev exponent, $1<q \leq p^{*}=p N /(N-p)$ is the critical Sobolev exponent, $f \in L^{\infty}\left(\mathcal{R}^{N}\right), \mathrm{h}$ is a bounded positive function on $\mathcal{R}^{k}$ and $\lambda$ is a parameter that we will specify later..

When $\mathrm{k}=\mathrm{N}, \mu=0$ and $\mathrm{p}=2$. The fact that the number of positive solutions of equation $\left(\mathcal{P}_{\lambda, \mu}\right)$ is affected by the nonlinearity terms which has been the focus of a great deal of research in recent years. If the weight functions $\mathrm{f} \equiv \mathrm{h} \equiv 1$, the authors Ambrosetti-Brezis-Cerami [1] have investigated

Corresponding author: Mohamed El Mokhtar ould El Mokhtar. E-mail: med.mokhtar66@yahoo.fr, M.labdi@qu.edu.sa. equation $\left(\mathcal{P}_{\lambda, \mu}\right)$. They found that there exists $\mu_{0}>0$ such that equation $\left(\mathcal{P}_{1, \mu}\right)$ admits at least two positive solutions for $0<\mu<\mu_{0}$, and has a positive solution for $\mu=\mu_{0}$ but no positive solution exists for $\mu>\mu_{0}$. For more general results, were done by de Figueiredo-Grossez-Ubilla [2], $\mathrm{Wu}$ [3], Cao etal. [4], Filippucci et al. [5], Xuan et al. [6], Guo and Niu [7] and the references therein.

In the case of $1<\mathrm{k}<\mathrm{N}$, equations with cylindrical potentials were also studied by many people [8-14]. For instance, in [15], Xuan studied the multiple weak solutions for $\mathrm{p}$-Laplace equation with singularity and cylindrical symmetry in bounded domains. However, they only considered the equation with sole critical Hardy-Sobolev term.

Let $\mathcal{D}_{1}^{p}\left(\mathcal{R}^{N}\right)$ be the space defined as the completion of $\mathcal{C}_{C}^{\infty}\left(\mathcal{R}^{N}\right)$ with respect to the norm

$$
\|\nabla u\|_{p}=\left(\int_{\mathcal{R}^{N}}|\nabla u|^{p} d x\right)^{\frac{1}{p}} .
$$

Clearly, the problem $\left(\mathcal{P}_{\lambda, \mu}\right)$ is related to the following Hardy-Sobolev type inequality with cylidrical weight which first proved in [10]

$\int_{\mathcal{R}^{N}}|\nabla u|^{p} d x \geq C \int_{\mathcal{R}^{N}}|y|^{-s}|u|^{p^{*}(s)} d x$, for all $u \in \mathcal{D}_{1}^{p}\left(\mathcal{R}^{N}\right)(1)$ where $\mathrm{C}>0,1<\mathrm{p}<\mathrm{k}, 2<\mathrm{k}<\mathrm{N}, \mathrm{x}=(\mathrm{y}, \mathrm{z}) \in$ 
$\mathcal{R}^{k} \times \mathcal{R}^{N-k}, \quad 0<\mathrm{s}<\mathrm{p}, \quad p^{*}(\mathrm{~s})=\mathrm{p}(\mathrm{N}-\mathrm{s}) /(\mathrm{N}-\mathrm{p})$, $p^{*}(s)=\mathrm{pN} /(\mathrm{N}-\mathrm{p}), 1<\mathrm{q}<\mathrm{p}$. In particular, for $\mathrm{s}=\mathrm{p}$ and $1<\mathrm{p}<\mathrm{k}$, we have Hardy type inequality:

$$
\int_{\mathcal{R}^{N}}|\nabla u|^{p} d x \geq \bar{\mu}_{k, p} \int_{\mathcal{R}^{N}}|y|^{-p}|u|^{p} d x \text {, for all } u \in \mathcal{D}_{1}^{p}\left(\mathcal{R}^{N}\right)(2)
$$

the constant $\bar{\mu}_{k, p}:=((k-p) / p)^{p}$ is sharp but not achieved [10].

When $\mu<\bar{\mu}_{k, p}$, Hardy type inequality implies that the norm

$$
\|u\|=\|u\|_{\mu, p}=\left(\int_{\mathcal{R}^{N}}\left(|\nabla u|^{p}-\mu|y|^{-p}|u|^{p}\right) d x\right)^{1 / p},
$$

is will defined in $\mathcal{D}_{1}^{p}\left(\mathcal{R}^{N}\right)$ and $\|$.$\| is equivalent to$ $\|\nabla .\|_{p}$; since the following inequalities hold:

$$
\begin{aligned}
& \left(1-\left(\max (\mu, 0) / \bar{\mu}_{k, p}\right)\right)^{1 / p}\|\nabla u\|_{p} \\
\leq & \|u\| \leq\left(1-\left(\min (\mu, 0) / \bar{\mu}_{k, p}\right)\right)^{1 / p}\|\nabla u\|_{p}
\end{aligned}
$$

for all $\mathrm{u} \in \mathcal{D}_{1}^{p}\left(\mathcal{R}^{N}\right)$.

Since our approach is variational, we define the functional $J_{\lambda}$ on $\mathcal{D}_{1}^{p}\left(\mathcal{R}^{N}\right)$ by

$$
J_{\lambda}(u):=(1 / p)\|u\|^{p}-\left(1 / p^{*}(s)\right) P(u)-(\lambda / q) Q(u),
$$

With

$$
P(u):=\int_{\mathcal{R}^{N}}|y|^{-s} h|u|^{*^{*}(s)} d x, Q(u):=\int_{\mathcal{R}^{N}} f|u|^{q} d x .
$$

Let

$$
S=S_{(\mu, N, p, 0)}:=\inf _{u \in \mathcal{D}_{1}^{p}\left(\mathcal{R}^{N}\right) \backslash\{0\}} \frac{\|u\|^{p}}{\left(\int_{\mathcal{R}^{N}}|u|^{p^{*}} d x\right)^{p / p^{*}}}
$$

and

$$
\tilde{S}=S_{(\mu, N, p, s)}:=\inf _{u \in \mathcal{D}_{1}^{p}\left(\mathcal{R}^{N}\right) \backslash\{0\}} \frac{\|u\|^{p}}{\left(\int_{\mathcal{R}^{N}|l|}||^{-s}|u|^{p^{*}(s)} d x\right)^{p / p^{*}(s)}}(4)
$$

where $0<\mathrm{s}<\mathrm{p}$. From [10], $\tilde{S}$ is achieved.

Throughout this work, we consider the following assumption:

(H) $\lim _{|y| \rightarrow 0} h(y)=\lim _{|y| \rightarrow \infty} h(y)=h_{0}>0, h(y) \geq h_{0}, y \in \mathcal{R}^{k}$.

In our work, we research the critical points as the minimizers of the energy functional associated to the problem $\left(\mathcal{P}_{\lambda, \mu}\right)$ on the constraint defined by the Nehari manifold, which are solutions of our problem.

Let $\Lambda_{0}$ be positive number

$$
\Lambda_{0}:=L(p, q)(\tilde{S})^{p^{*}(s)\left(p^{*}-p\right) / p\left(p-p^{*}(s)\right)}(S)^{-p^{*} / q}
$$

where

$$
\begin{aligned}
L(p, q): & {\left[\left(\frac{p-p^{*}(s)}{\left(q-p^{*}(s)\right)|f|_{\infty}}\right)\right] \times } \\
& {\left[|h|_{\infty}\left(\frac{p^{*}(s)-q}{(p-q)}\right)\right]^{\left(p^{*}-p\right) /\left(p^{*}(s)-p\right)} }
\end{aligned}
$$

and $|f(x)|_{\infty}=\sup _{x \in \mathcal{R}^{N}}|f(x)|,|h(y)|_{\infty}=\sup _{y \in \mathcal{R}^{k}}|h(y)|$.

Now we can state our main results.

Theorem 1: Let $\mathrm{f} \in L^{\infty}\left(\mathcal{R}^{N}\right)$. Assume that $1<\mathrm{p}<\mathrm{k}$, $\mathrm{N}>\mathrm{p}, 2<\mathrm{k}<\mathrm{N}, 0<\mu<\bar{\mu}_{k, p}:=((k-p) / p)^{p}, 0$ $<\mathrm{s}<\mathrm{p}, 1<\mathrm{q}<\mathrm{p}$, (H) satisfied and $\lambda$ verifying $0<\lambda<\Lambda_{0}$, then the equation $\left(\mathcal{P}_{\lambda, \mu}\right)$ has at least one positive solution.

Theorem 2: In addition to the assumptions of the Theorem 1, there exists a positive real $\Lambda_{1}$ such that, if $\lambda$ satisfy $0<\lambda<\Lambda_{2}=\min \left(\Lambda_{0}, \Lambda_{1}\right), \quad$ then $\left(\mathcal{P}_{\lambda, \mu}\right)$ has at least two positive solutions.

Theorem 3: In addition to the assumptions of the Theorem 2, there exists a positive real $\Lambda_{*}$ such that, if $\lambda$ satisfy $0<\lambda<\min \left(\Lambda_{2}, \Lambda_{*}\right)$, then $\left(\mathcal{P}_{\lambda, \mu}\right)$ has at least two positive solution and two opposite solutions.

Theorem 4: Let $\mathrm{f} \in L^{\infty}\left(\mathcal{R}^{N}\right)$. Assume that $1<\mathrm{p}$ $<\mathrm{k}, \mathrm{N}>\mathrm{p}, 0<\mathrm{s}<\mathrm{p}, \mu<0, q=p^{*}$, (H) satisfied and $\lambda>0$, then the problem $\left(\mathcal{P}_{\lambda, \mu}\right)$ has a nontrivial cylindrical weak solution $\mathrm{u} \in X_{l}\left(\mathcal{R}^{N}\right)(\mathrm{u}$ satisfying $\mathrm{u}(\mathrm{y}, \mathrm{z})=\mathrm{u}(|\mathrm{y}|, \mathrm{z}))$.

This paper is organized as follows. In Section 2, we give some preliminaries. Section 3 is devoted to the proofs of Theorems 1, 2, 3 and 4.

\section{Preliminaries}

Definition 1: Let $\mathrm{c} \in \mathcal{R}, \mathrm{E}$ a Banach space and $J_{\lambda} \in C^{1}(E, \mathcal{R})$.

(i) $\left(u_{n}\right)_{n}$ is a Palais-Smale sequence at level c ( in short $(P S)_{c}$ ) in E for $J_{\lambda}$ if

$$
J_{\lambda}\left(u_{n}\right)=c+o_{n}(1) \text { and } J_{\lambda}^{\prime}\left(u_{n}\right)=o_{n}(1),
$$

where $O_{n}(1)$ tends to 0 as $n$ goes at infinity.

(ii) We say that $J_{\lambda}$ satisfies the $(P S)_{C}$ condition if any $(P S)_{c}$ sequence in $\mathrm{E}$ for $J_{\lambda}$ has a convergent 
subsequence.

Lemma 1: Let $X$ Banach space, and $J_{\lambda} \in C^{1}(X, \mathcal{R})$ verifying the Palais -Smale condition. Suppose that $J_{\lambda}(0)=0$ and that:

(i) there exist $\mathrm{R}>0, \mathrm{r}>0$ such that if \|\|$u\|\|=\mathrm{R}$, then $J_{\lambda}(\mathrm{u}) \geq \mathrm{r}$;

(ii) there exist $\mathrm{u}_{0} \in \mathrm{X}$ such that $\left\|\mathrm{u}_{\mathrm{o}}\right\|>\mathrm{R}$ and $J_{\lambda}$ $\left(\mathrm{u}_{\mathrm{o}}\right) \leq 0$;

let $c=\inf _{\gamma \in \Gamma} \max _{t \in[0,1]}\left(J_{\lambda}(\gamma(t))\right)$

$\Gamma=\left\{\begin{array}{c}\gamma \in C([0,1] ; X) \text { such that } \\ \gamma(0)=0 \text { et } \gamma(1)=u_{0}\end{array}\right\}$

then c is critical value of $J_{\lambda}$ such that $\mathrm{c} \geq \mathrm{r}$.

\subsection{Nehari Manifold}

It is well known that $J_{\lambda}$ is of class $C^{1}$ in $\mathcal{D}_{1}^{p}\left(\mathcal{R}^{N}\right)$ and the solutions of $\left(\mathcal{P}_{\lambda, \mu}\right)$ are the critical points of $J_{\lambda}$ which is not bounded below on $\mathcal{D}_{1}^{p}\left(\mathcal{R}^{N}\right)$. Consider the following Nehari manifold

$$
\mathcal{N}=\left\{u \in \mathcal{H} \backslash\{0\}:\left\langle J_{\lambda}^{\prime}(u), u\right\rangle=0\right\},
$$

Thus, $\mathrm{u} \in \mathrm{N}$ if and only if

$$
\|u\|^{p}-P(u)-\lambda Q(u)=0
$$

Note that $\mathrm{N}$ contains every nontrivial solution of the problem $\left(\mathcal{P}_{\lambda, \mu}\right)$. Moreover, we have the following results.

Lemma 2: $J_{\lambda}$ is coercive and bounded from below on $\mathrm{N}$.

Proof If $\mathrm{u} \in \mathrm{N}$, then by Eq. (5) and the Sobolev inequality, we deduce that

$$
\begin{aligned}
J_{\lambda}(u)= & \left(\left(p^{*}(s)-p\right) / p p^{*}(s)\right)\|u\|^{p} \\
& -\lambda\left(\left(p^{*}(s)-q\right) / q p^{*}(s)\right) Q(u, v) \\
\geq & \left(\left(p^{*}(s)-p\right) / p p^{*}(s)\right)\|u\|^{p} \\
& -\lambda\left(\left(p^{*}(s)-q\right) / q p^{*}(s)\right)|f|_{\infty} S^{\left(q / p^{*}\right)}\|u\|^{q}
\end{aligned}
$$

Thus, $J_{\lambda}$ is coercive and bounded from below on $\mathrm{N}$. Define

Then, for $\mathrm{u} \in \mathrm{N}$

$$
\begin{aligned}
\left\langle\phi^{\prime}(u), u\right\rangle & =p\|u\|^{p}-p^{*}(s) P(u)-\lambda q Q(u) \\
& =(p-q)\|u\|^{p}-\left(p^{*}(s)-q\right) P(u) \\
& =\lambda\left(p^{*}(s)-q\right) Q(u)-\left(p^{*}(s)-p\right)\|u\|^{p}
\end{aligned}
$$

Now, we split $\mathrm{N}$ in three parts:

$$
\begin{aligned}
& \mathcal{N}^{+}=\left\{u \in \mathcal{N}:\left\langle\phi^{\prime}(u), u\right\rangle>0\right\} \\
& \mathcal{N}^{0}=\left\{u \in \mathcal{N}:\left\langle\phi^{\prime}(u), u\right\rangle=0\right\} \\
& \mathcal{N}^{-}=\left\{u \in \mathcal{N}:\left\langle\phi^{\prime}(u), u\right\rangle<0\right\}
\end{aligned}
$$

We have the following results.

Lemma 3: Suppose that $\mathrm{u}_{0}$ is a local minimizer for $J_{\lambda}$ on $\mathrm{N}$. Then, if $\mathrm{u}_{0} \notin \mathrm{N}^{0}, \mathrm{u}_{0}$ is a critical point of $J_{\lambda}$.

Proof If $\mathrm{u}_{0}$ is a local minimizer for $J_{\lambda}$ on $\mathrm{N}$, then $\mathrm{u}_{0}$ is a solution of the optimization problem

$$
\min _{\{u / \phi(u)=0\}} J_{\lambda}(u) .
$$

Hence, there exists a Lagrange multipliers $\theta \in \mathrm{R}$ such that

$$
J_{\lambda}^{\prime}\left(u_{0}\right)=\theta \phi^{\prime}\left(u_{0}\right) \text { in }\left(\mathcal{D}_{1}^{p}\left(\mathcal{R}^{N}\right)\right)^{\prime}
$$

Thus,

$$
\left\langle J_{\lambda}^{\prime}\left(u_{0}, v_{0}\right),\left(u_{0}, v_{0}\right)\right\rangle=\theta\left\langle\phi^{\prime}\left(u_{0}, v_{0}\right),\left(u_{0}, v_{0}\right)\right\rangle \text {. }
$$

But $\left\langle\phi^{\prime}\left(u_{0}\right), u_{0}\right\rangle \neq 0$, since $u_{0} \notin N^{0}$. Hence $\theta=0$. This completes the proof.

Lemma 4: There exists a positive number $\Lambda_{0}$ such that for all verifying $0<\lambda<\Lambda_{0}$, we have $\mathrm{N}^{0}=\emptyset$.

Proof Let us reason by contradiction. Suppose $\mathrm{N}^{0} \neq$ $\emptyset$ such that $0<\lambda<\Lambda_{0}$, Then, by Eq. (7) and for $\mathrm{u}$ $\in \mathrm{N}^{\mathrm{o}}$, we have

$$
\begin{aligned}
\|u\|^{p} & =\left(p^{*}(s)-q\right) /(p-q) P(u, v) \\
& =\lambda\left(\left(p^{*}(s)-q\right) /\left(p^{*}(s)-p\right)\right) Q(u, v)
\end{aligned}
$$

Moreover, by the Holder inequality and the Sobolev embedding theorem, we obtain

$$
\|u\| \geq(S)^{p^{*} / q\left(p^{*}-p\right)}\left[\left(p-p^{*}(s)\right) / \lambda\left(q-p^{*}(s)\right)|f|_{\infty}\right]^{-1 /\left(p^{*}-p\right)}(9)
$$
and

$$
\|u\| \leq\left[h_{0}\left(\left(p^{*}(s)-q\right) /(p-q)\right)\right]^{1 /\left(p-p^{*}(s)\right)}(\tilde{S})^{-p^{*}(s) / p\left(p--p^{*}(s)\right)}(10)
$$

From Eq. (9) and Eq. (10), we obtain $\lambda \geq \Lambda_{0}$, which contradicts an hypothesis. 
Thus $\mathrm{N}=\mathrm{N}^{+} \cup \mathrm{N}^{-}$. Define

$$
c:=\inf _{u \in \mathcal{N}} J_{\lambda}(u), c^{+}:=\inf _{u \in \mathcal{N}^{+}} J_{\lambda}(u) \text { and } c^{-}:=\inf _{u \in \mathcal{N}^{-}} J_{\lambda}(u) .
$$

For the sequel, we need the following Lemma.

Lemma 5:

(i) For all such that $0<\lambda<\Lambda_{0}$, one has $\mathrm{c} \leq \mathrm{c}^{+}<0$.

(ii) There exists $\Lambda_{1}>0$ such that for all $0<\lambda<\Lambda_{2}=\min \left(\Lambda_{0}, \Lambda_{1}\right)$ one has

$$
\begin{gathered}
c^{-}>C_{0}=C_{0}\left(\lambda, p, q, S, \tilde{S}, p^{*}(s), h_{0},|f|_{\infty}\right) \\
=\left(\frac{\left(p^{*}(s)-p\right)}{p p^{*}(s)}\right)\left[\frac{(p-q)}{\left(p^{*}(s)-q\right) h_{0}}\right]^{\frac{-p}{\left(p-p^{*}(s)\right)}}(\tilde{S})^{\frac{p^{*}(s)}{\left(p^{*}(s)-p\right)}}+ \\
\quad-\lambda\left(\frac{\left(p^{*}(s)-q\right)}{q\left(p^{*}(s)\right)}\right)|f|_{\infty}(S)^{q / p^{*}} .
\end{gathered}
$$

Proof (i) Let $u \in N^{+}$. By Eq. (7), we have

$$
\left[(p-q) /\left(p^{*}(s)-q\right)\right]\|u\|^{p}>P(u)
$$

and so

$$
\begin{aligned}
J_{\lambda}(u) & =(-(p-q) / p q)\|u\|^{p}+\left(\left(p^{*}(s)-q\right) / q\left(p^{*}(s)\right)\right) P(u) \\
& <-\left[((p-q) / p q)+\left((p-q) / q\left(p^{*}(s)\right)\right)\right]\|u\|^{p} .
\end{aligned}
$$

We conclude that $\mathrm{c} \leq \mathrm{c}^{+}<0$.

(ii) Let $\mathrm{u} \in \mathrm{N}^{-}$. By Eq. (7), we get

$$
\left[(p-q) /\left(p^{*}(s)-q\right)\right]\|u\|^{p}<P(u) .
$$

Moreover, by $(\mathrm{H})$ and Sobolev embedding theorem, we have

$$
P(u) \leq(\tilde{S})^{-p^{*}(s) / p}\left|h^{+}\right|_{\infty}\|u\|^{p^{*}(s)} .
$$

This implies

$$
\|u\|>(\tilde{S})^{p^{*}(s) / p\left(p^{*}(s)-p\right)}\left[\frac{(p-q)}{\left(p^{*}(s)-q\right)\left|h^{+}\right|_{\infty}}\right]^{\frac{-1}{\left(p-p^{*}(s)\right)}} \text {, for all } u \in \mathcal{N}^{-}(11)
$$

By Eq. (6), we get

$$
\begin{aligned}
J_{\lambda}(u) \geq & \left(\frac{\left(p^{*}(s)-p\right)}{p p^{*}(s)}\right)\left[\frac{(p-q)}{\left(p^{*}(s)-q\right) h_{0}}\right]^{\frac{-p}{\left(p-p^{*}(s)\right)}}(\tilde{S})^{\frac{p^{*}(s)}{\left(p^{*}(s)-p\right)}}+ \\
& -\lambda\left(\frac{\left(p^{*}(s)-q\right)}{q\left(p^{*}(s)\right)}\right)|f|_{\infty}(S)^{q / p^{*}} .
\end{aligned}
$$

Thus, for all $\lambda$ such that $0<\lambda<\Lambda_{2}=\min \left(\Lambda_{0}, \Lambda_{1}\right)$, with

$$
\begin{aligned}
\Lambda_{1}: & =\left(\frac{\left(p^{*}(s)-p\right)}{p p^{*}(s)}\right)\left[\frac{(p-q)(\tilde{S})^{\frac{p^{*}(s)}{p}}}{\left(p^{*}(s)-q\right) h_{0}}\right]^{\frac{-p}{\left(p-p^{*}(s)\right)}} \\
& \times\left[\left(\frac{\left(p^{*}(s)-q\right)|f|_{\infty}}{q\left(p^{*}(s)\right)}\right)(S)^{-q / p^{*}}\right]^{-1}
\end{aligned}
$$

we have $J_{\lambda} \geq \mathrm{C}_{0}$.
For each $\mathrm{u} \in \mathcal{D}_{1}^{p}\left(\mathcal{R}^{N}\right)$, we write

$$
t_{m}:=t_{\max }(u)=\left[\frac{\|u\|^{p}}{\left(p^{*}(s)-q\right) P(u)}\right]^{1 /\left(p^{*}(s)-p\right)}>0 .
$$

Lemma 5: Let real parameters such that $0<\lambda<\Lambda_{0}$. For each $\mathrm{u} \in \mathcal{D}_{1}^{p}\left(\mathcal{R}^{N}\right)$, one has the following:

(i) If $\mathrm{Q}(\mathrm{u}) \leq 0$, then there exists a unique $\mathrm{t}^{-}>t_{m}$ such that $\mathrm{t}^{-} \mathrm{u} \in \mathrm{N}^{-}$and

$$
J_{\lambda}\left(t^{-} u\right)=\sup _{t \geq 0}(t u) .
$$

(ii) If $\mathrm{Q}(\mathrm{u})>0$, then there exist unique $\mathrm{t}^{+}$and $\mathrm{t}^{-}$such that $0<\mathrm{t}^{+}<t_{m} \quad<\mathrm{t}^{-},\left(\mathrm{t}^{+} \mathrm{u}\right) \in \mathrm{N}^{+},\left(\mathrm{t}^{-} \mathrm{u}\right) \in \mathrm{N}^{-}$,

$$
J_{\lambda}\left(t^{+} u\right)=\inf _{0 \leq t \leq t_{m}} J_{\lambda}(t u) \text { and } J_{\lambda}\left(t^{-} u\right)=\sup _{t \geq 0} J_{\lambda}(t u) \text {. }
$$

Proof With minor modifications, we refer to [16].

Proposition 1 [6]

(i) For all such that $0<\lambda<\Lambda_{0}$, there exists a $(P S)_{C^{+}}$sequence in $\mathrm{N}^{+}$.

(ii) For all such that $0<\lambda<\Lambda_{2}=\min \left(\Lambda_{0}, \Lambda_{1}\right)$, there exists a a $(P S)_{C^{-}}$sequence in $\mathrm{N}^{-}$.

\section{Proof of Theorems}

\subsection{Proof of Theorem 1}

Now, taking as a starting point the work of Tarantello [17], we establish the existence of a local minimum for $J_{\lambda}$ on $\mathrm{N}^{+}$.

Proposition 2 For all such that $0<\lambda<\Lambda_{0}$, the functional $J_{\lambda}$ has a minimizer $\mathrm{u}_{0}{ }^{+} \in \mathrm{N}^{+}$and it satisfies:

(i) $J_{\lambda}\left(\mathrm{u}_{0}^{+}\right)=\mathrm{c}=\mathrm{c}^{+}$,

(ii) $\mathrm{u}_{0}{ }^{+}$is a nontrivial solution of $\left(\mathcal{P}_{\lambda, \mu}\right)$.

Proof If $0<\lambda<\Lambda_{0}$, then by Proposition1 (i), there exists a $\left(u_{n}\right)_{n}(P S)_{c^{+}}$sequence in $\mathrm{N}^{+}$, thus, it bounded by Lemma 4 . Then, there exists $\mathrm{u}_{0}{ }^{+} \in$ $\mathcal{D}_{1}^{p}\left(\mathcal{R}^{N}\right)$ and we can extract a subsequence which will denoted by $\left(u_{n}\right)_{n}$ such that

$$
\begin{aligned}
& u_{n} \rightarrow u_{0}^{+} \text {weakly in } \mathcal{D}_{1}^{p}\left(\mathcal{R}^{N}\right) \\
& u_{n} \rightarrow u_{0}^{+} \text {weakly in }\left(L^{p^{*}(s)}\left(\mathcal{R}^{N},|y|^{-s}\right)\right) \\
& u_{n} \rightarrow u_{0}^{+} \text {strongly in } L^{q}\left(\mathcal{R}^{N}\right) \\
& u_{n} \rightarrow u_{0}^{+} \text {a.e in } \mathcal{R}^{N}
\end{aligned}
$$


Thus, by Eq. (12), $\mathrm{u}_{0}{ }^{+}$is a weak nontrivial solution of $\left(\mathcal{P}_{\lambda, \mu}\right)$. Now, we show that $\left(u_{n}\right)_{n}$ converges to $\mathrm{u}_{0}{ }^{+}$strongly in $\mathcal{D}_{1}^{p}\left(\mathcal{R}^{N}\right)$. Suppose otherwise. By the lower semi-continuity of the norm, then $\left\|u_{0}^{+}\right\|<\liminf _{n \rightarrow \infty}\left\|u_{n}\right\|$ and we obtain

$$
\begin{aligned}
c \leq & J_{\lambda}\left(u_{0}^{+}\right)=\left(\left(p^{*}(s)-p\right) / p\left(p^{*}(s)\right)\right)\left\|u_{0}^{+}\right\|^{p} \\
& -\left(\left(p^{*}(s)-q\right) / q\left(p^{*}(s)\right)\right) Q\left(u_{0}^{+}\right) \\
< & \liminf _{n \rightarrow \infty} J\left(u_{n}\right)=c .
\end{aligned}
$$

We get a contradiction. Therefore, $\left(u_{n}\right)_{n}$ converge to $\mathrm{u}_{0}{ }^{+}$strongly in $\mathcal{D}_{1}^{p}\left(\mathcal{R}^{N}\right)$. Moreover, we have $\mathrm{u}_{0}{ }^{+} \in \mathrm{N}^{+}$. If not, then by Lemma 5 , there are two numbers $\mathrm{t}_{0}{ }^{+}$and $\mathrm{t}_{0}{ }^{-}$, uniquely defined so that $\left(\mathrm{t}_{0}{ }^{+} \mathrm{u}_{0}{ }^{+}\right) \in$ $\mathrm{N}^{+}$and $\left(\mathrm{t}^{-} \mathrm{u}_{0}{ }^{+}\right) \in \mathrm{N}^{-}$. In particular, we have $\mathrm{t}_{0^{+}}{ }^{+}<\mathrm{t}_{0}{ }^{-}=1$. Since

$$
\frac{d}{d t} J_{\lambda}\left(t u_{0}^{+}\right)_{\mid t=t_{0}^{+}}=0 \text { and } \frac{d^{2}}{d t^{2}} J_{\lambda}\left(t u_{0}^{+}\right)_{\mid t=t_{0}^{+}}>0,
$$

there exists $\mathrm{t}_{0}{ }^{+}<\mathrm{t}^{-} \leq \mathrm{t}_{0}{ }^{-}$such that $J_{\lambda}\left(\mathrm{t}_{0}{ }^{+} \mathrm{u}_{0}{ }^{+}\right)<J_{\lambda}$ $\left(\mathrm{t}^{-} \mathrm{u}_{0}{ }^{+}\right)$. By Lemma 5, we get

$$
J_{\lambda}\left(t_{0}^{+} u_{0}^{+}\right)<J_{\lambda}\left(t^{-} u_{0}^{+}\right)<J_{\lambda}\left(t_{0}^{-} u_{0}^{+}\right)=J_{\lambda}\left(u_{0}^{+}\right),
$$

which contradicts the fact that $J_{\lambda}\left(\mathrm{u}_{0}{ }^{+}\right)=\mathrm{c}^{+}$. Since $J_{\lambda}\left(\mathrm{u}_{0}{ }^{+}\right)=J_{\lambda}\left(\left|\mathrm{u}_{0}{ }^{+}\right|\right)$and $\left|\mathrm{u}_{0}{ }^{+}\right| \in \mathrm{N}^{+}$, then by Lemma 2 , we may assume that $\mathrm{u}_{0}{ }^{+}$is a nontrivial nonnegative solution of $\left(\mathcal{P}_{\lambda, \mu}\right)$. By the Harnack inequality, we conclude that $\mathrm{u}_{0}{ }^{+}>0$ and $\mathrm{v}_{0}{ }^{+}>0$, see for example [18].

\subsection{Proof of Theorem 2}

Next, we establish the existence of a local minimum for on $\mathrm{N}^{-}$. For this, we require the following Lemma.

Lemma 6 For all such that $0<\lambda<\Lambda_{2}=\min \left(\Lambda_{0}, \Lambda_{1}\right)$, the functional $J_{\lambda}$ has a minimizer $\mathrm{u}_{0}{ }^{-}$in $\mathrm{N}^{-}$and it satisfies:

(i) $J_{\lambda}\left(u_{0}^{-}\right)=c^{-}>0$,

(ii) $u_{0}^{-}$is a nontrivial solution of $\left(\mathcal{P}_{\lambda, \mu}\right)$ in $\mathcal{D}_{1}^{p}\left(\mathcal{R}^{N}\right)$.

Proof If $0<\lambda<\Lambda_{2}=\min \left(\Lambda_{0}, \Lambda_{1}\right)$, then by Proposition 1 (ii) there exists a $\left(u_{n}\right)_{n},(P S)_{c^{-}}$ sequence in $\mathrm{N}^{-}$, thus it bounded by Lemma 1. Then, there exists $u_{0}{ }^{-} \in \mathcal{D}_{1}^{p}\left(\mathcal{R}^{N}\right)$ and we can extract a subsequence which will denoted by $\left(u_{n}\right)_{n}$ such that

$$
\begin{aligned}
& u_{n} \rightarrow u_{0}^{-} \text {weakly in } \mathcal{D}_{1}^{p}\left(\mathcal{R}^{N}\right) \\
& u_{n} \rightarrow u_{0}^{-} \text {weakly in } L^{p^{*}(s)}\left(\mathcal{R}^{N},|y|^{-s}\right) \\
& u_{n} \rightarrow u_{0}^{-} \text {strongly in } L^{q}\left(\mathcal{R}^{N}\right) \\
& u_{n} \rightarrow u_{0}^{-} \text {a.e in } \mathcal{R}^{N}
\end{aligned}
$$

This implies

$$
P\left(u_{n}\right) \rightarrow P\left(u_{0}^{-}\right) \text {, as } n \text { goes to } \infty \text {. }
$$

Moreover, by $(\mathrm{H})$ and Eq. (7) we obtain

$$
P\left(u_{n}\right)>(p-q) /\left(p^{*}(s)-q\right)\left\|u_{n}\right\|^{p}
$$

By Eq. (9) and Eq. (13) there exists a positive number

$$
C_{1}:=\left[(p-q) /\left(p^{*}(s)-q\right)\right]^{p^{*}(s) /\left(p^{*}(s)-p\right)}(\tilde{S})^{p^{*}(s) /\left(p^{*}(s)-p\right)},
$$

such that

$$
P\left(u_{n}\right)>C_{1}
$$

This implies that

$$
P\left(u_{0}^{-}\right) \geq C_{1} .
$$

Now, we prove that $\left(u_{n}\right)_{n}$ converges to $u_{0}{ }^{-}$ strongly in $\mathcal{D}_{1}^{p}\left(\mathcal{R}^{N}\right)$. Suppose otherwise. Then, ॥ $\left\|u_{0}^{-}\right\|<\liminf _{n \rightarrow \infty}\left\|u_{n}\right\|$

\|. By Lemma 5 there is a unique $\mathrm{t}_{0}{ }^{-}$such that $\left(\mathrm{t}_{0}{ }^{-} \mathrm{u}_{0}{ }^{-}\right) \in \mathrm{N}^{-}$. Since

$$
u_{n} \in \mathcal{N}^{-}, J_{\lambda}\left(u_{n}\right) \geq J_{\lambda}\left(t u_{n}\right), \text { for all } t \geq 0,
$$

we have

$$
J_{\lambda}\left(t_{0}^{-} u_{0}^{-}\right)<\lim _{n \rightarrow \infty} J_{\lambda}\left(t_{0}^{-} u_{n}\right) \leq \lim _{n \rightarrow \infty} J_{\lambda}\left(u_{n}\right)=c^{-},
$$

and this is a contradiction. Hence,

$$
u_{n} \rightarrow u_{0}^{-} \text {strongly in } \mathcal{D}_{1}^{p}\left(\mathcal{R}^{N}\right) .
$$

Thus,

$J_{\lambda}\left(u_{n}\right)$ converges to $J_{\lambda}\left(u_{0}^{-}\right)=c^{-}$as $n$ tends to $+\infty$.

Since $J_{\lambda}\left(\mathrm{u}_{0}^{-}\right)=J_{\lambda}\left(\left|\mathrm{u}_{0}^{-}\right|\right)$and $\mathrm{u}_{0}^{-} \in \mathrm{N}^{-}$, then by Eq. (14) and Lemma 2, we may assume that $u_{0}{ }^{-}$is a nontrivial nonnegative solution of $\left(\mathcal{P}_{\lambda, \mu}\right)$. By the maximum principle, we conclude that $\mathrm{u}_{0}{ }^{-}>0$ and $\mathrm{v}_{0}{ }^{-}>0$.

Now, we complete the proof of Theorem 2. By Proposition 2 and Lemma 6, we obtain that $\left(\mathcal{P}_{\lambda, \mu}\right)$ has two positive solutions $\mathrm{u}_{0}^{+} \in \mathrm{N}^{+}$and $\mathrm{u}_{0}{ }^{-} \in \mathrm{N}^{-}$. Since $\mathrm{N}^{+} \cap \mathrm{N}^{-}=\varnothing$, this implies that $\mathrm{u}_{0}{ }^{+}$and $\mathrm{u}_{0}{ }^{-}$are distinct. 


\subsection{Proof of Theorem 3}

In this section, we consider the following Nehari submanifold of $\mathrm{N}$

$$
\mathcal{N}_{\varrho}=\left\{\begin{array}{c}
u \in \mathcal{D}_{1}^{p}\left(\mathcal{R}^{N}\right) \backslash\{0\}:\left\langle J_{\lambda}^{\prime}(u), u\right\rangle=0 \\
\text { and }\|u\| \geq \varrho>0
\end{array}\right\}
$$

Thus, $\mathrm{u} \in \mathcal{N}_{\varrho}$ if and only if

$$
\|u\|^{p}-P(u)-\lambda Q(u)=0 \text { and }\|u\| \geq \varrho>0
$$

Firstly, we need the following Lemmas

Lemma 7 Under the hypothesis of theorem 3, there exist $\varrho_{0}, \Lambda_{3}>0$ such that $\mathcal{N}_{\varrho}$ is nonempty for any $\lambda \in\left(0, \Lambda_{3}\right)$ and $\varrho \in\left(0, \varrho_{0}\right)$.

Proof Fix $\left(\mathrm{u}_{0}\right) \in \mathcal{D}_{1}^{p}\left(\mathcal{R}^{N}\right) \backslash\{0\}$ and let

$$
\begin{aligned}
g(t) & =\left\langle J_{\lambda}^{\prime}\left(t u_{0}\right), t u_{0}\right\rangle \\
& =t^{p}\left\|u_{0}\right\|^{p}-t^{p^{*}(s)} P\left(u_{0}\right)-t \lambda Q\left(u_{0}\right)
\end{aligned}
$$

Clearly $\mathrm{g}(0)=0$ and $\mathrm{g}(\mathrm{t}) \rightarrow-\infty$ as $\mathrm{n} \rightarrow+\infty$. Moreover, we have

$$
\begin{aligned}
& g(1)=\left\|u_{0}\right\|^{p}-P\left(u_{0}\right)-\lambda Q\left(u_{0}\right) \\
& \geq\left[\left\|u_{0}\right\|^{p}-(\tilde{S})^{-p^{*}(s) / p}\left|h^{+}\right|_{\infty}\left\|u_{0}\right\|^{p^{*}(s)}\right]-\lambda\left\|u_{0}\right\| \\
& \text { If }\left\|u_{0}\right\| \geq \varrho>0 \text { for } \\
& 0<\varrho<\varrho_{0}:=\left(\left|h^{+}\right|_{\infty}\left(p^{*}(s)-1\right)\right)^{\frac{-1}{\left(p^{*}(s)-p\right)}}(\tilde{S})^{\frac{p^{*}(s)}{p\left(p^{*}(s)-p\right)}}, \\
& \left|h^{+}\right|_{\infty} \in\left(0, \alpha_{0}\right) \text { with } \\
& \alpha_{0}:=(\tilde{S})^{p^{*}(s) / p} /\left(\left(p^{*}(s)-1\right)\right)^{\left(p^{*}(s)-1\right) / p^{*}(s)}
\end{aligned}
$$
and

then, there exists

$$
\Lambda_{3}:=\left[\left(\left|h^{+}\right|_{\infty}\left(p^{*}(s)-1\right)\right)(\tilde{S})^{-p^{*}(s) / p}\right]^{-1 /\left(p^{*}(s)-p\right)}-\Theta \times \Phi
$$

where

$$
\Theta:=\left(\left(p^{*}(s)-1\right)\right)^{p^{*}(s)-1}\left(\left(\left|h^{+}\right|_{\infty}\right)^{p^{*}(s) / p}(\tilde{S})\right)^{-(2 *)^{2} / 2}
$$

and

$$
\Phi:=\left[\left(\left.h^{+}\right|_{\infty}\left(p^{*}(s)-1\right)\right)(\tilde{S})^{-p^{*}(s) / p}\right]^{-1 /\left(p^{*}(s)-p\right)}
$$

and there exists $\mathrm{t}_{0}>0$ such that $\mathrm{g}\left(\mathrm{t}_{0}\right)=0$. Thus, $\mathrm{t}_{0} \mathrm{u}_{0} \in$ $\mathcal{N}_{\varrho}$ and $\mathcal{N}_{\varrho}$ is nonempty for any $\lambda \in\left(0, \Lambda_{3}\right)$.

Lemma 8 There exist $M, \Lambda_{*}$ positive reals such that

$$
\left\langle\phi^{\prime}(u), u\right\rangle<-M<0, \text { for } u \in \mathcal{N}_{\varrho},
$$

and any $\lambda$ verifying

$$
0<\lambda<\min \left(\Lambda_{2}, \Lambda_{*}\right) .
$$

Proof Let $\mathrm{u} \in \mathcal{N}_{\varrho}$, then by Eq. (5) and Eq. (7), it allows us to write

$$
\begin{aligned}
\left\langle\phi^{\prime}(u), u\right\rangle & =\left(p-p^{*}(s)\right)\|u\|^{p}+\lambda\left(p^{*}(s)-q\right) Q(u) \\
& \leq\left(p-p^{*}(s)\right)\|u\|^{p}+\lambda\left(p^{*}(s)-q\right)\left|f^{+}\right|_{\infty} S^{q / p^{*}(s)}\|u\|^{q} \\
& \leq \max \left(\|u\|^{p},\|u\|^{q}\right)\left[\left(p-p^{*}(s)\right)+\lambda\left(p^{*}(s)-q\right)\left|f^{+}\right|_{\infty} S^{q / p^{*}(s)}\right]
\end{aligned}
$$

Thus, for any $\lambda$ verifying

$0<\lambda<\Lambda_{4}=\left[\frac{\left(p^{*}(s)-p\right)}{\left(p^{*}(s)-q\right)\left|f^{+}\right|_{\infty}}\right] S^{q / p^{*}(s)}$,

and choosing $\Lambda_{*}=\min \left(\Lambda_{3}, \Lambda_{4}\right)$ with $\Lambda_{3}$

defined in Lemma 1, then we obtain that

$$
\left\langle\phi^{\prime}(u), u\right\rangle<0 \text {, for any } u \in \mathcal{N}_{\varrho}
$$

Lemma 9 Suppose $\mathrm{N}>\mathrm{p}, 0<\mathrm{s}<\mathrm{p}, 1<\mathrm{q}<2$. Then, there exist $\mathrm{r}$ and positive constants $\eta$ such that

(i) we have $J_{\lambda}(u) \geq \eta>0$ for $\|u\|=r$.

(ii) there exists $\sigma \in \mathcal{N}_{\varrho}$ when $\|\sigma\|>r$, with $r=\|u\| \|$, such that $J_{\lambda}(\sigma) \leq 0$.

Proof We can suppose that the minima of $J_{\lambda}$ are realized by $\mathrm{u}_{0}{ }^{+}$and $\mathrm{u}_{0}{ }^{-}$. The geometric conditions of the mountain pass theorem are satisfied. Indeed, we have

(i) By Eq. (7) and Eq. (15), we get

$$
\begin{aligned}
J_{\lambda}(u) \geq[ & \left.\left(p^{*}(s)-p\right) / p p^{*}(s)\right]\|u\|^{p} \\
& -\left[\left(p^{*}(s)-p\right) / q p^{*}(s)\right]\|u\|^{p+q},
\end{aligned}
$$

Thus, there exist $\eta, \mathrm{r}>0$ such that

$$
J_{\lambda}(u) \geq \eta>0 \text { when } r=\|u\| \text { small. }
$$

(ii) Let $\mathrm{t}>0$, then we have for all $\phi \in \mathcal{N}_{\varrho}$

$$
J_{\lambda}(t \phi):=\left(t^{p} / p\right)\|\phi\|^{p}-\left(t^{p^{*}(s)}\right) P(\phi)-\lambda\left(t^{q} / q\right) Q(\phi) \text {. }
$$

Letting $\sigma=t \phi$ for $\mathrm{t}$ large enough. Since $P(\phi)>0$,

we obtain $J_{\lambda}(\sigma) \leq 0$.For $\mathrm{t}$ large enough we can ensure $\|\sigma\|>r$.

Let $\Gamma$ and $\mathrm{c}$ defined by

$$
\Gamma:=\left\{\gamma:[0,1] \rightarrow \mathcal{N}_{\varrho}: \gamma(0)=u_{0}^{-} \text {and } \gamma(1)=u_{0}^{+}\right\}
$$

and

$$
c:=\inf _{\gamma \in \Pi} \max _{t \in[0,1]}\left(J_{\lambda}(\gamma(t))\right) .
$$


Proof of Theorem 3:

If $0<\lambda<\min \left(\Lambda_{2}, \Lambda_{*}\right)$ then, by the Lemma 4 and Proposition 1 (ii), $J_{\lambda}$ verifying the Palais -Smale condition in $\mathcal{N}_{\varrho}$. Moreover, from the Lemmas 3, 8 and 9 , there exists $u_{c}$ such that

$$
J_{\lambda}\left(u_{c}\right)=c \text { and } u_{c} \in \mathcal{N}_{\varrho} \text {. }
$$

Thus $u_{c}$ is the third solution of our system such that $u_{c} \neq \mathrm{u}_{0}{ }^{+}$and $u_{c} \neq \mathrm{u}_{0}{ }^{-}$. Since $\left(\mathcal{P}_{\lambda, \mu}\right)$ is odd with respect $\mathrm{u}$, we obtain that $\left(-u_{C}\right)$ is also a solution of $\left(\mathcal{P}_{\lambda, \mu}\right)$.

\subsection{Proof of Theorem 4}

In the part, we consider the case $\mu<0, q=p^{*}$ and $\lambda>0$ and obtain the existence of the solution with cylidrical symmetry for $\left(\mathcal{P}_{\lambda, \mu}\right)$. First, we list some notations.

Define

$$
\begin{gathered}
X:=X\left(\mathcal{R}^{N} ;|y|^{-p} d x\right):= \\
\left\{u \in \mathcal{D}_{1}^{p}\left(\mathcal{R}^{N}\right): \int_{\mathcal{R}^{N}}|y|^{-p} h u^{p} d x<+\infty\right\} \\
X_{l}:=X_{l}\left(\mathcal{R}^{N} ;|y|^{-p} d x\right):= \\
\{u \in X: u(y, z)=u(|y|, z)\}
\end{gathered}
$$

Now, we set $E(u)$ as the energy functional of equation $\left(\mathcal{P}_{\lambda, \mu}\right)$ that is

$$
\begin{aligned}
E(u): & =(1 / p) \int_{\mathcal{R}^{N}}|\nabla u|^{p} d x-(\mu / p) \int_{\mathcal{R}^{N}}|y|^{-p}|u|^{p} d x \\
& -\left(1 / p^{*}(s)\right) P(u)-\left(\lambda / p^{*}\right) Q(u),
\end{aligned}
$$

With

$$
P(u):=\int_{\mathcal{R}^{N}}|y|^{-s} h|u|^{p^{*}(s)} d x, Q(u):=\int_{\mathcal{R}^{N}} f|u|^{p^{*}} d x .
$$

The functional $\mathrm{E}(\mathrm{u})$ is belong to $C^{1}\left(X, \mathcal{R}^{N}\right)$. Following, we can define a group of rescaling operators:

$$
T_{\eta, x} u:=\eta^{-\left(\frac{N-p}{p}\right)} u\left(\eta^{-1} \cdot+x\right) .
$$

By direct computation, we have

$$
T_{\eta, x} u:=T_{\frac{1}{\eta},-\eta x} u, T_{\eta_{1}, x_{1}} T_{\eta_{2}, x_{2}} u:=T_{\eta_{1} \eta_{2}, x_{1}+x_{2}} u
$$

and if $\mathrm{u} \in L^{p^{*}}\left(\mathcal{R}^{N}\right)$ and $\mathcal{D}_{1}^{p}\left(\mathcal{R}^{N}\right)$, one get $T_{\eta, x} u \in L^{p^{*}}\left(\mathcal{R}^{N}\right)$ and $\mathcal{D}_{1}^{p}\left(\mathcal{R}^{N}\right)$. We know that the mapping $u \in L^{p^{*}}\left(\mathcal{R}^{N}\right) \mapsto L^{p^{*}}\left(\mathcal{R}^{N}\right)$ and $u \in \mathcal{D}_{1}^{p}\left(\mathcal{R}^{N}\right) \mapsto T_{\eta, x} u \in \mathcal{D}_{1}^{p}\left(\mathcal{R}^{N}\right)$ are isometric. As the method we used here is the concentration-compactness principle, and some propositions in [8], we list them first:

Lemma 10 (the concentration-compactness principle of Solimini ) If $\left(u_{k}\right) \subset \mathcal{D}_{1}^{p}\left(\mathcal{R}^{N}\right)$ is bounded, then up to a subsequence, $\left(u_{k}\right)$ converge strongly to 0 in $L^{p^{*}}\left(\mathcal{R}^{N}\right)$ or there exists $\left(\eta_{k}\right) \subset(0,+\infty)$ and $\left(x_{k}\right) \subset \mathcal{R}^{N}$ such that $T_{\eta_{k}, x_{k}} u_{k} \rightarrow u$ in $L^{p^{*}}\left(\mathcal{R}^{N}\right), \mathrm{u} \neq 0$.

Proposition 3 [8] Let $1<\gamma<\infty$, assuming $\left(\eta_{k}\right) \subset(0,+\infty)$ and $\left(x_{k}\right) \subset \mathcal{R}^{N}$ are such that $\eta_{k} \rightarrow \eta, x_{k} \rightarrow x$, then

$$
\begin{gathered}
T_{\eta_{k}, x_{k}} u_{k} \rightarrow T_{\eta, x} u \text { in } L^{\gamma}\left(\mathcal{R}^{N}\right) \\
\text { if } u_{k} \rightarrow u \text { in } L^{\gamma}\left(\mathcal{R}^{N}\right) .
\end{gathered}
$$

Proposition 4 If $u \in X_{l}$ then for all $\psi \in X$ and $\mathrm{g} \in \mathrm{O}(\mathrm{k})$, we have $E^{\prime}(u) \psi(g .,)=.E^{\prime}(u) \psi$, where $\mathrm{O}(\mathrm{k})$ is the orthogonal group of $\mathcal{R}^{k}$.

The proof is similar to the proof of Proposition 10 in [8], we omit it.

By a similar analysis in Proposition 3, we get that for the functional $\left.E\right|_{X_{l}}$, there exist a bounded sequence $\left(x_{k}\right) \subset X_{l}$ and $\mathrm{c}>0$ such that

$$
E\left(u_{k}\right) \rightarrow c \text { and }\left.E^{\prime}\left(u_{k}\right)\right|_{X_{l}} \rightarrow 0 \text { in } X_{l}^{\prime} \text { (dual of } X_{l} \text { ). }
$$
where $\mathrm{c}$ is the mountain pass level of $\left.E\right|_{X_{l}}$ defined by

$$
\begin{aligned}
c= & \inf _{\delta \in \Gamma} \max _{t \in[0,1]} E(\delta(t)), \Gamma:= \\
& \left\{\delta \in\left([0,1], X_{l}\right): \delta(0)=0, E(\delta(1))<0\right\}
\end{aligned}
$$

Now we begin to prove Theorem 4. Since the sequence $\left(u_{k}\right)$ is bounded, it satisfies one of the cases in Lemma 10, now we show that the first case doesn't occur.

Lemma 11 The case $\left(u_{k}\right) \rightarrow 0$ in $L^{p^{*}}\left(\mathcal{R}^{N}\right)$ doesn't hold.

Proof If not, then

$$
\begin{aligned}
\int_{\mathcal{R}^{N}}|y|^{-s}\left|u_{k}\right|^{p^{*}(s)} d x & \leq \int_{\mathcal{R}^{N}}|y|^{-s}\left|u_{k}\right|^{p}\left|u_{k}\right|^{p^{*}(s)-p} d x \\
& \leq\left(\int_{\mathcal{R}^{N}}|y|^{-\frac{s N}{N-p+s}}\left|u_{k}\right|^{\frac{p N}{N-p+s}} d x\right)^{\frac{N-p+s}{N}}\left(\int_{\mathcal{R}^{N}}\left|u_{k}\right|^{p^{*}} d x\right)^{\frac{p^{*}(s)-p}{p^{*}}}(16) \\
& \leq C\left(\int_{\mathcal{R}^{N}}\left|\nabla u_{k}\right|^{p} d x\right)\left(\int_{\mathcal{R}^{N}}\left|u_{k}\right|^{p^{*}} d x\right)^{\frac{p^{*}(s)-p}{p^{*}}}
\end{aligned}
$$


Hence, when $\left(u_{k}\right) \rightarrow 0$ in $L^{p^{*}}\left(\mathcal{R}^{N}\right)$, we have

$$
\int_{\mathcal{R}^{N}}|y|^{-s}\left|u_{k}\right|^{p^{*}(s)} d x \rightarrow 0 \text {. }
$$

Now, in fact that $\mu<0$ and

$$
\begin{gathered}
E^{\prime}\left(u_{k}\right) u_{k}=\int_{\mathcal{R}^{N}}\left|\nabla u_{k}\right|^{p} d x-\mu \int_{\mathcal{R}^{N}}|y|^{-p}\left|u_{k}\right|^{p} d x \\
-\int_{\mathcal{R}^{N}}|y|^{-s} h\left|u_{k}\right|^{p^{*}(s)} d x-\int_{\mathcal{R}^{N}} f\left|u_{k}\right|^{p^{*}} d x \rightarrow 0,
\end{gathered}
$$

one get

$$
\int_{\mathcal{R}^{N}}\left|\nabla u_{k}\right|^{p} d x \rightarrow 0 \text { and } \int_{\mathcal{R}^{N}}|y|^{-p}\left|u_{k}\right|^{p} d x \rightarrow 0 .
$$

Then, we obtain

$$
\begin{array}{r}
E\left(u_{k}\right)=(1 / p) \int_{\mathcal{R}^{N}}\left|\nabla u_{k}\right|^{p} d x-(\mu / p) \int_{\mathcal{R}^{N}}|y|^{-p}\left|u_{k}\right|^{p} d x \\
\left(1 / p^{*}(s)\right) \int_{\mathcal{R}^{N}}|y|^{-s} h\left|u_{k}\right|^{p^{*}(s)} d x-\left(1 / p^{*}\right) \int_{\mathcal{R}^{N}} f\left|u_{k}\right|^{p^{*}} d x \rightarrow 0 .
\end{array}
$$

It contradict the fact that $E\left(u_{k}\right) \rightarrow c>0$. Therefore, Lemma $\mathbf{1 1}$ is proved.

As conclusion, by Lemma 10 and Lemma 11, one has that there exists $\left(u_{k}\right) \subset(0,+\infty)$ and $\left(x_{k}\right) \subset \mathcal{R}^{N}$ such that

$$
T_{\eta_{k}, x_{k}} u_{k} \rightarrow u \text { in } L^{p^{*}}\left(\mathcal{R}^{N}\right), u \neq 0
$$

Setting $x_{k}=\left(y_{k}, z_{k}\right)=\bar{y}_{k}+\bar{z}_{k}$

where

$$
\bar{y}_{k}=\left(y_{k}, 0\right), \bar{z}_{k}=\left(0, z_{k}\right)
$$

and

$$
y_{k} \in \mathcal{R}^{d}, z_{k} \in \mathcal{R}^{N-d} .
$$

Defining $v_{k}:=T_{\eta_{k}, \bar{z}_{k}} u_{k}$, we get

Lemma 12 The sequence $\left(v_{k}\right)$ is bounded in $X_{l}$ and it satisfies

$$
E\left(v_{k}\right) \rightarrow c, E^{\prime}\left(v_{k}\right) \rightarrow 0 \text { in } X_{l}^{\prime}
$$

and

$$
v_{k}\left(.+\eta_{k}, \bar{y}_{k}\right) \rightarrow u \text { in } L^{p^{*}}\left(\mathcal{R}^{N}\right)
$$

Proof Since $\left(u_{k}\right)$ is bounded in $X_{l}$ and the operators $T_{\eta_{k}, \bar{z}_{k}}$ are isometries of $X_{l}$, we get $\left(v_{k}\right)$ is bounded in $X_{l}$ easily. By Eq. (17) we obtain the formula (18).

Now, we say that

$$
E\left(u_{k}\right)=E\left(v_{k}\right) \text { and }\left\|E^{\prime}\left(v_{k}\right)\right\|_{X_{l}^{\prime}}=\left\|E^{\prime}\left(u_{k}\right)\right\|_{X_{l}^{\prime}} \text {. }
$$

In fact, one has

$$
\begin{aligned}
E\left(v_{k}\right): & (1 / p) \int_{\mathcal{R}^{N}}\left|\nabla v_{k}\right|^{p} d x-(\mu / p) \int_{\mathcal{R}^{N}}|y|^{-p}\left|v_{k}\right|^{p} d x \\
& -\left(1 / p^{*}(s)\right) \int_{\mathcal{R}^{N}}|y|^{-s} h\left|v_{k}\right|^{p^{*}(s)} d x-\left(1 / p^{*}\right) \int_{\mathcal{R}^{N}} f\left|v_{k}\right|^{p^{*}} d x \\
= & (1 / p) \int_{\mathcal{R}^{N}}\left|\nabla u_{k}\right|^{p} d x-(\mu / p) \int_{\mathcal{R}^{N}}|y|^{-p}\left|u_{k}\right|^{p} d x \\
& -\left(1 / p^{*}(s)\right) \int_{\mathcal{R}^{N}}|y|^{-s} h\left|u_{k}\right|^{p^{*}(s)} d x-\left(1 / p^{*}\right) \int_{\mathcal{R}^{N}} f\left|u_{k}\right|^{p^{*}} d x \\
= & E\left(u_{k}\right),
\end{aligned}
$$

and for all $\psi \in X_{l}^{\prime}$, we have

$$
\begin{aligned}
& \left\langle E^{\prime}\left(v_{k}\right), \psi\right\rangle \\
= & \left.\int_{\mathcal{R}^{N}} \nabla T_{\eta_{k}, \bar{z}_{k}} u_{k}\right|^{p-2} \nabla T_{\eta_{k}, \bar{z}_{k}} u_{k} \cdot \nabla \psi d x-\mu \int_{\mathcal{R}^{N}}|y|^{-p}\left|T_{\eta_{k}, \bar{z}_{k}} u_{k}\right|^{p-2} T_{\eta_{k}, \bar{z}_{k}} u_{k} \cdot \psi d x \\
& -\int_{\mathcal{R}^{N}}|y|^{-s} h\left|T_{\eta_{k}, \bar{z}_{k}} u_{k}\right|^{p^{*}(s)-2} T_{\eta_{k}, \bar{z}_{k}} u_{k} \cdot \psi d x-\left.\int_{\mathcal{R}^{N}} f T_{\eta_{k}, \bar{z}_{k}} u_{k} \cdot \psi\right|^{p^{*}-2} T_{\eta_{k}, \bar{z}_{k}} u_{k} \cdot \psi d x \\
= & \eta_{k}^{\frac{N-p}{p}} \int_{\mathcal{R}^{N}}\left|\nabla u_{k}\right|^{p-2} \nabla u_{k} \cdot \nabla \psi\left(\eta_{k} X-\eta_{k} \bar{z}_{k}\right) d x \\
& -\mu \eta_{k}^{\frac{N-p}{p}} \int_{\mathcal{R}^{N}}|y|^{-p}\left|u_{k}\right|^{p-2} u_{k} \cdot \psi\left(\eta_{k} x-\eta_{k} \bar{z}_{k}\right) d x \\
& -\eta_{k}^{\frac{N-p}{p}} \int_{\mathcal{R}^{N}}|y|^{-s} h\left|u_{k}\right|^{p^{*}(s)-2} u_{k} \cdot \psi\left(\eta_{k} X-\eta_{k} \bar{z}_{k}\right) d x \\
& -\eta_{k}^{\frac{N-p}{p}} \int_{\mathcal{R}^{N}} f\left|u_{k} \cdot \psi\right|^{p^{*}-2} u_{k} \cdot \psi\left(\eta_{k} X-\eta_{k} \bar{z}_{k}\right) d x \\
= & \left\langle E^{\prime}\left(u_{k}\right), T_{\eta_{k}^{-1},-,-\eta_{k} \bar{z}_{k}} \psi\right\rangle .
\end{aligned}
$$

So one get $\left\|E^{\prime}\left(v_{k}\right)\right\|_{X_{l}^{\prime}}=\left\|E^{\prime}\left(u_{k}\right)\right\|_{X_{l}^{\prime}}$.

Proposition 4 [8] Let $\left(\varphi_{m}\right) \subset \mathcal{R}^{k}$ such that $\lim _{m \rightarrow \infty}\left|\varphi_{m}\right|=+\infty, \mathrm{R}>0$ fixed, then for any $\mathrm{t} \in$ $\mathrm{N} \backslash\{0,1\}$ there exists $m_{t} \in \mathcal{N}$ such that for any $m>m_{t}$ one can find $g_{1}, \ldots, g_{t} \in O(k)$ satisfying the condition $i \neq j \Rightarrow B_{R}\left(g_{i} \varphi_{m}\right) \cap B_{R}\left(g j \varphi_{m}\right)=\emptyset$.

Lemma $13 \mathrm{Up}$ to a subsequence $\left(v_{k}\right)$, there exists $v \in X_{l}$ and $v \neq 0$ such that

$$
v_{k} \rightarrow v \text { in } X_{l} \text {. }
$$

Proof: since $\left(v_{k}\right)$ is bounded in $X_{l}$, we can assume that $v_{k} \rightarrow v$ in $X_{l}$, if $\mathrm{v}=0$, we will show contradiction. Indeed, from Eq. (17) we know that

$$
T_{1, \eta_{k} \bar{y}_{k}} \rightarrow v \text { in } L^{p^{*}}\left(\mathcal{R}^{N}\right) .
$$

To get contradiction, we first prove that

$$
\lim _{m \rightarrow \infty} \eta_{k} \bar{y}_{k}=+\infty
$$

If not, then up to a subsequence, $\lim _{m \rightarrow \infty} \eta_{k} \bar{y}_{k}=\bar{y}_{0}$. Therefore, Lemma 12 implies

$v_{k}=T_{1,0} v_{k}=T\left(T_{1,-\eta_{k} \bar{y}_{k}} T_{1,-\eta_{k} \bar{y}_{k}}\right) v_{k} \rightarrow T_{1,-\bar{y}_{0}} u \neq 0$, it contradicts our assumption $v_{k} \rightarrow v=0$.

Since $\mathrm{u} \neq 0$, there exist $\omega>0$ and $D \subseteq \mathcal{R}^{N}$ 
with $|\mathrm{D}| \neq 0$ such that either $u>\omega$ or $u<-\omega$ almost everywhere in $\mathrm{D}$. Given $\mathrm{R}>0$ such that $\left|B_{R} \cap D\right|>0$, by weak convergence we get

$$
\int_{\mathcal{R}^{N}} T_{1, \eta_{k} \bar{y}_{k}} v_{k} \chi_{B_{R} \cap D} d x \rightarrow \int_{\mathcal{R}^{N}} u \chi_{B_{R} \cap D} d x \geq \omega\left|B_{R} \cap D\right|>\text { (20) }
$$

On the other hand,

$$
\begin{aligned}
\left|\int_{\mathcal{R}^{N}} T_{1, \eta_{k} \bar{y}_{k}} v_{k} \chi_{B_{R} \cap D} d x\right| & \leq \int_{B_{R}}\left|T_{1, \eta_{k} \bar{y}_{k}} v_{k}\right| d x \\
& =\int_{B_{R}}\left|v_{k}\left(\left(x+\eta_{k} \bar{y}_{k}\right)\right)\right| d x \\
& =\int_{B_{R}\left(\eta_{k} \bar{y}_{k}\right)}\left|v_{k}(x)\right| d x \\
& \leq C\left(\int_{B_{R}\left(\eta_{k} \bar{y}_{k}\right)}\left|v_{k}(x)\right|^{p^{*}} d x\right)^{1 / p^{*}}
\end{aligned}
$$

where $\mathrm{C}$ only depends on $\mathrm{R}$ and $\mathrm{N}$. The relations of Eq. (20) and Eq. (21) imply that

$$
\lim \inf _{k \rightarrow \infty} \int_{B_{R}\left(\eta_{k} \bar{y}_{k}\right)}\left|v_{k}(x)\right|^{p^{*}} d x>0 .
$$

Up to a subsequence, we can assume that for some $\varepsilon>0$,

$$
\inf _{k} \int_{B_{R}\left(\eta_{k} \bar{y}_{k}\right)}\left|v_{k}(x)\right|^{p^{*}} d x>\varepsilon .
$$

Then, from Proposition 4, we have that for any $t \in$ $\mathrm{N} \backslash\{0,1\}$ and $m>m_{t}$

$$
\begin{aligned}
\int_{\mathcal{R}^{N}}\left|v_{k}(x)\right|^{p^{*}} d x & \geq \sum_{i=1}^{t} \int_{B_{R}\left(\eta_{k}\left(g_{t} \bar{y}_{k} .0\right)\right)}\left|v_{k}(x)\right|^{p^{*}} d x \\
& =\sum_{i=1}^{t} \int_{B_{R}\left(\eta_{k} \bar{y}_{k}\right)}\left|v_{k}(x)\right|^{p^{*}} d x \geq t \varepsilon
\end{aligned}
$$

This implies that $\left\|v_{k}\right\|_{L^{p^{*}}\left(\mathcal{R}^{N}\right)} \rightarrow \infty$, which contradicts the fact that $\left(v_{k}\right)$ is bounded in $L^{p^{*}}\left(\mathcal{R}^{N}\right)$

Proof of Theorem 4 From Lemmas 11 and 13, we get $\quad E^{\prime}\left(v_{k}\right) \rightarrow 0$ in $X_{l}^{\prime}$ and $v_{k} \rightarrow v \neq 0$ in $X_{l}\left(\mathcal{R}^{N}\right)$, which implies that $\mathrm{v}$ is a nontrivial cylindrical weak solution to the problem $\left(\mathcal{P}_{\lambda, \mu}\right)$.

\section{Conclusions}

In our work, we have searched the critical points as the minimizers of the energy functional associated to the problem $\left(\mathcal{P}_{\lambda, \mu}\right)$ on the constraint defined by the Nehari manifold, which are solutions of our problem.
In the sections 3 , we have proved the existence of at least four positive solutions by using a Nehari and sub-Nehari manifold and mountain pass theorem. In Section 3.4, we have considered the case $\mu<0, q=p^{*}$ and $\lambda>0$ and we have obtained the existence of the solution with cylidrical symmetry for $\left(\mathcal{P}_{\lambda, \mu}\right)$ on the space

$$
\begin{aligned}
X:=X\left(\mathcal{R}^{N} ;|y|^{-p} d x\right):= \\
\quad\left\{u \in \mathcal{D}_{1}^{p}\left(\mathcal{R}^{N}\right): \int_{\mathcal{R}^{N}}|y|^{-p} h u^{p} d x<+\infty\right\}
\end{aligned}
$$

by using the concentration-compactness principle.

\section{References}

[1] Ambrosetti, A., Brezis, H., and Cerami, G. 1994. "Combined effects of concave and convex nonlinearities in some elliptic problems.” J. Funct. Anal. 122: 519-543.

[2] de Figueiredo, D. G., Gossez, J. P., and Ubilla, P. 2003. "Local Superlinearity and Sublinearity for Indefinite Semilinear Elliptic Problems." J. Funct. Anal. 199: 452-467.

[3] Wu, T. F. "Multiplicity Results for a Semilinear Elliptic Equation Involving Sign-Changing Weight Function." Rocky Mountain Journal of Mathematics, in press.

[4] Cao, D. M., Peng, S. J., and Yan, S. S. 2012. "Infinitely Many Solutions for p-Laplacian Equation Involving Critical Sobolev Growth." $J$ Funct Anal. 262: 2861-2902.

[5] Filippucci, R., Pucci, P., and Robert, F. 2009. "On a p-Laplace Equation with Multiple Critical Nonlinearities.” J Math Pure Appl. 91: 156-177.

[6] Xuan, B. J., and Wang, J. 2010. "Existence of a Nontrivial Weak Solution to Quasilinear Elliptic Equations with Singular Weights and Multiple Critical Exponents." Nonlinear Analysis 72: 3649-3658.

[7] Li, Y. Y., Guo, Q. Q., and Niu, P. C. 2011. "Global Compactness Results for Quasilinear Elliptic Problems with Combined Critical Sobolev-Hardy Terms." Nonlinear Analysis. 74: 1445-1464.

[8] Badiale, M., Bergio, V., and Rolando, S. 2007. "A Nonlinear Elliptic Equation with Singular Potential and Applications to Nonlinear Field Equations." J Eur Math Soc. 9: 355-381.

[9] Badiale, M., Guida, M., and Rolando, S. 2007. "Elliptic Equations with Decaying Cylindrical Potentials and Power-Type Nonlinearities.” Adv Diff Equ. 12: 1321-1362.

[10] Badiale, M., and Tarantello, G. 2002. "A Sobolev-Hardy Inequality with Applications to a Nonlinear Elliptic Equation Arising in Astrophysics." Arch Ration Mech 
Anal. 163: 252-293.

[11] Bhakta, M., and Sandeep, K. 2009. "Hardy-Sobolev-Maz'ya Type Equations in Bounded Domains." J Differ Equ. 247: 119-139.

[12] Gazzini, M., Ambrosetti, A., and Musina, R. Nonlinear elliptic problems related to some integral inequalities. http://www.digitallibrary.sissa.it/retrieve/4320/PhDThesis Gazzini.pdf

[13] Gazzini, M., and Mussina, R. 2009. "On a Sobolev Type Inequality Related to the Weighted p-Laplace Operator." $J$ Math Anal Appl. 352: 99-111.

[14] Musina, R. 2008. "Ground State Solutions of a Critical Problem Involving Cylindrical Weights.” Nonlinear Anal. 68: 3972-3986.
[15] Xuan, B. J. 2003. "Multiple Solutions to p-Laplacian Equation with Singularity and Cylindrical Symmetry." Nonlinear Analysis 55: 217-232.

[16] Brown, K. J., and Zhang, Y. 2003. "The Nehari Manifold for a Semilinear Elliptic Equation with a Signchanging Weight Function.” Journal of Differential Equations 193: 481-499.

[17] Tarantello, G. 1993. "Multiplicity Results for an Inhomogeneous Neumann Problem Critical Exponent." Manuscripta Math. 81: 57-78.

[18] Drabek, P., Kufner, A., and Nicolosi, F. 1997. "Quasilinear Elliptic Equations with Degenerations and Singularities." Walter de Gruyter Series in Nonlinear Analysis and Applications 5 (New York, 1997). 